\title{
A Time-Domain Model for the Study of High-Frequency Wheelset-Track Interaction
}

\author{
Wenshan Fang ${ }^{1}$ (1) $\cdot$ José Martinez-Casas $^{2} \cdot$ Stefano Bruni $^{1}$
}

Received: 7 October 2016/Revised: 16 December 2016/ Accepted: 16 January 2017/Published online: 7 February 2017

(C) The Author(s) 2017. This article is published with open access at Springerlink.com

\begin{abstract}
A mathematical model of dynamic wheelset-track interaction is proposed in this paper. The model is defined in the time domain in order to introduce and correctly evaluate nonlinear and time-variant phenomena related to the contact model and boundary conditions which play a very important role in rail surface degradation phenomena. The complete model can be divided into three main components: the model of the wheelset, the model of the track and the model of wheel-rail contact forces. In the paper, the wheelset is described as a rotating flexible body, and the gyroscopic and inertial effects associated with wheelset rotation are introduced to this model using an 'Eulerian' finite element approach based on 3D quadratic solid elements. The discrete supported track is modelled using finite Timoshenko beam element, which takes into account both the vertical and the lateral rail vibration valid up to $1500 \mathrm{~Hz}$. The wheelset and the track are coupled by means of a contact model based on the nonlinear Hertz and Kalker theories. The flexible components of the interaction model make it possible to describe the train-track dynamics in a relatively high-frequency range, which allows the investigation of specific aspects such as rail corrugation. Some numerical results are presented in terms of contact forces and rail-wheel vibration speed in the paper. The effect of wheelset and track flexibility in specific frequency range on train-track interaction dynamics is briefly discussed.
\end{abstract}

Wenshan Fang

wenshan.fang@polimi.it

1 Mechanical Engineering Department, Politecnico di Milano, 20156 Milan, Italy

2 Centro de Investigación en Ingeniería Mecánica, Universitat Politècnica de València, Camino de Vera s/n, 46022 Valencia, Spain

Editor: Xuesong Zhou
Keywords Flexible wheelset · Flexible track · Train-track interaction $\cdot$ High-frequency dynamics

\section{Introduction}

Train-track interaction has been extensively studied in the last 40 years at least, leading to modelling approaches that can deal satisfactorily with many dynamic problems arising at the wheel-rail interface. Some important dynamic phenomena, for instance, rolling noise [1] and vehicle-track coupled vibration response [2] caused by the roughness of the wheel and rail running surfaces have been investigated with frequency-domain wheel-rail interaction models, where the dynamic response characteristics at wheel-rail contact are derived by combining the wheelset and track frequency response functions with the assumed rail roughness [3]. However, frequency-domain method gets its limit when comes to nonlinear components in wheel-rail contact and vehicle suspension. To take consideration of the effect of nonlinearities in the track and in wheel-rail contact, train-track interaction models in time domain are widely studied today: in [4-14], the train-track interaction is defined in time domain with the vehicle and/or the track model defined using the finite element method.

In state-of-the-art papers, different configurations of the vehicle model and the track model are used to investigate the dynamic influences of a train running along a track under specific targets. To investigate the track dynamics, detailed track model coupled with relatively simple wheelset model was used. Zhai et al. [9] investigated the influence of elastic track structures on the lateral hunting stability of different railway vehicles. Baeza et al. [10] developed a cyclic boundary track model based on modal substructuring. Di Gialleonardo et al. [13] investigated the 
effect of different levels of track flexibility on running behaviours of both tangent and curved tracks. To investigate the wheelset dynamics, efforts have been made to develop detailed rotating flexible wheelset model. Arnold et al. [5] and Kaiser et al. [7, 13], proposed the use of a semi-analytical solution of a rotational symmetric structure in two dimensions, using Navier's equations. Baeza et al. [8, 11], on the other hand, modelled the rotating wheelset based on Brown and Shabana's formulation [15, 16] of kinematics of flexible rotating structure using a Lagrangian coordinate. However, open problems still exist related to wheel-rail interaction in the high-frequency range and its relationship with wheelset flexibility. Furthermore, the effect of wheelset and track flexibility at different levels still needs to be investigated in full.

The aim of this paper is to define a wheelset-track interaction model valid in a frequency range up to $1500 \mathrm{~Hz}$ and hence suitable for the investigation of typical problems related to high-frequency train-track interaction such as short-pitch rail corrugation. To this aim, a new coupling of a full rotating flexible wheelset model together with a discrete supported Timoshenko finite element track model is developed. The wheelset model is developed using a Eulerian approach [14]. The equations of wheelset motion are deducted using virtual work principal, and the convergence of the model is guaranteed using integral by parts, which has not been treated in previous work. The track model describes displacement and rotation of both vertical and lateral directions. The results of this new model are presented by comparing the running behaviours under different model configurations.

\section{Mathematic Model of the Wheelset-Track Interaction}

The vehicle model used in this paper considers one single wheelset and the primary suspension only. The second suspension system is ignored, with the bogie frame and the car body masses represented by two static forces applied through the primary suspension on the two sides. The reason of this simplification is due to the mechanical filter effect introduced by the suspensions: the dynamics of the sprung masses of vehicle (bogie frame and car body) occur in low-frequency area under $20 \mathrm{~Hz}$ and thus are well isolated from the high-frequency vibrations of wheelsets and can consequently be simplified as lumped masses $[10,11,14,17]$ when modelling the high-frequency vehicle-track interaction.

\subsection{Model of the Rotating Flexible Wheelset}

When modelling the rotating flexible wheelset, the interested contact points are always applied to the interface with the rail. The external forces of the wheelset are applied in the direction and displacement of the contact points, which is a fixed spatial point. Thus, a Eulerian approach originally from [14] is introduced to describe the kinematic motion of the wheelset. The position vector $\mathbf{r}$ is defined as:

$\mathbf{r}=\mathbf{u}+\mathbf{w}(\mathbf{u}, t)$,

where $\mathbf{u}$ is the position of an undeformed spatial point and $\mathbf{w}(\mathbf{u}, t)$ is the displacement associated with wheelset flexibility.

The wheelset is set to rotate with spin velocity $\Omega$ in the second axle, and the angular velocity tensor verifies:

$\tilde{\mathbf{\Omega}}=\left[\begin{array}{ccc}0 & 0 & \Omega \\ 0 & 0 & 0 \\ -\Omega & 0 & 0\end{array}\right]=\Omega \mathbf{J} ; \quad$ and $\quad \mathbf{J} \cdot \mathbf{J}=-\mathbf{E} ;$

where $J$ and $E$ are defined as:

$\mathbf{J}=\left[\begin{array}{ccc}0 & 0 & 1 \\ 0 & 0 & 0 \\ -1 & 0 & 0\end{array}\right] ; \quad \mathbf{E}=\left[\begin{array}{lll}1 & 0 & 0 \\ 0 & 0 & 0 \\ 0 & 0 & 1\end{array}\right]$

The spin velocity of the wheelset is:

$\mathbf{V}=\left(\begin{array}{lll}v_{1} & v_{2} & v_{3}\end{array}\right)^{\mathrm{T}}=\tilde{\mathbf{\Omega}} \mathbf{u}=\Omega \mathbf{J} \mathbf{u}=\Omega \tilde{\mathbf{u}}$

where $\tilde{\mathbf{u}}=\left(\begin{array}{lll}u_{3} & 0 & -u_{1}\end{array}\right)^{\mathrm{T}}$.

The equation of wheelset motion is formulated by virtual work principle. The velocity and acceleration of the particle are computed through the material derivatives as follows:

$$
\begin{aligned}
\dot{\mathbf{r}} & =\frac{D \mathbf{r}}{D t}=\frac{D \mathbf{u}}{D t}+\frac{D \mathbf{w}}{D t}=\sum_{i=1}^{3} v_{i} \frac{\partial \mathbf{u}}{\partial u_{i}}+\dot{\mathbf{w}}+\sum_{i=1}^{3} v_{i} \frac{\partial \mathbf{w}}{\partial u_{i}} \\
& =\Omega \mathbf{J u}+\dot{\mathbf{w}}+\Omega \sum_{i=1}^{3} \tilde{\mathbf{u}}_{\mathbf{i}} \frac{\partial \mathbf{w}}{\partial u_{i}} ;
\end{aligned}
$$

$\ddot{\mathbf{r}}=\frac{D^{2} \mathbf{r}}{D t^{2}}$

$$
\begin{aligned}
= & \Omega \mathbf{J} \frac{\partial u}{\partial t}+\frac{\partial \dot{\mathbf{w}}}{\partial t}+\sum_{i=1}^{3} v_{\mathbf{i}} \frac{\partial \dot{\mathbf{w}}}{\partial u_{i}}+\Omega \sum_{i=1}^{3} \frac{\partial \tilde{u}_{i}}{\partial t} \frac{\partial \mathbf{w}}{\partial u_{i}} \\
& +\Omega \sum_{i=1}^{3} \tilde{u}_{i} \frac{\partial^{2} \mathbf{w}}{\partial u_{i}^{2}} \frac{\partial u_{i}}{\partial t}+\Omega \sum_{i=1}^{3} \tilde{u}_{i} \frac{\partial \dot{\mathbf{w}}}{\partial u_{i}}
\end{aligned}
$$

The virtual work associated with the inertial forces is:

$$
\begin{aligned}
\delta W= & \int_{\mathrm{Vol}} \rho \delta \mathbf{w}^{\mathrm{T}} \ddot{r} \mathrm{~d} v=\int_{\mathrm{Vol}} \rho \delta \mathbf{w}^{\mathrm{T}} \ddot{\mathbf{w}} \mathrm{d} v+2 \Omega \int_{\mathrm{Vol}} \rho \delta \mathbf{w}^{\mathrm{T}} \sum_{i=1}^{3} \tilde{u}_{i} \frac{\partial \dot{\mathbf{w}}}{\partial u_{i}} \mathrm{~d} v \\
& +\Omega^{2}\left(-\int_{\mathrm{Vol}} \rho \delta \mathbf{w}^{\mathrm{T}} \sum_{i=1}^{3} \mathbf{E} u_{i} \frac{\partial \mathbf{w}}{\partial u_{i}} \mathrm{~d} v\right. \\
& \left.+\int_{\mathrm{Vol}} \rho \delta \mathbf{w}^{\mathrm{T}} \sum_{i=1}^{3} \sum_{j=1}^{3} \tilde{u}_{i} \tilde{u}_{j} \frac{\partial^{2} \mathbf{w}}{\partial u_{i} \partial u_{j}} \mathrm{~d} v-\int_{\mathrm{Vol}} \rho \delta \mathbf{w}^{\mathrm{T}} \sum_{i=1}^{3} \mathbf{E} u_{i} \mathrm{~d} v\right)
\end{aligned}
$$

The virtual work equation is then solved by finite element integration. In this step, the integrals in the volume of 
the solid are computed as the sum of integrals on each volume of the finite element elements:

$\int_{\mathrm{Vol}} \cdot \mathrm{d} v=\sum_{\text {elements }} \int_{V^{\mathrm{e}}} \cdot \mathrm{d} v$

To reduce the computational cost, a modal approach and nodal interpolation are adopted after the finite element integration. The displacement of the $e$ th element in volume $V^{\mathrm{e}}$ is:

$\mathbf{w}(\mathbf{u}, t)=\mathbf{N}^{\mathrm{e}}(\mathbf{u}) \mathbf{w}^{\mathrm{e}}(t)=\mathbf{N}^{\mathrm{e}}(\mathbf{u}) \Phi^{\mathrm{e}}(\mathbf{u}) \mathbf{q}(t) \quad \mathbf{u} \in V^{\mathrm{e}}$

where $\mathbf{N}^{\mathrm{e}}(\mathbf{u})$ is the shape function matrix of the $e$ th element, $\mathbf{w}^{\mathrm{e}}(t)$ is the nodal displacement of the $e$ th element, $\boldsymbol{\Phi}^{\mathrm{e}}(\mathbf{u})$ is the mode shapes of the eth element computed from the finite element model of the wheelset, and $\mathbf{q}(t)$ is the modal coordinate vector. Introduce Eq. (9) into Eq. (7), one can obtain:

$$
\begin{aligned}
& \delta W=\sum_{e=1}^{\text {Nelements }} \delta \mathbf{q}^{\mathrm{eT}} \Phi_{F E}^{\mathrm{eT}} \\
& \times\left(\begin{array}{c}
\int_{V^{\mathrm{e}}} \rho \mathbf{N}^{\mathrm{eT}} \mathbf{N}^{\mathrm{e}} \mathrm{d} v \ddot{\mathbf{q}}^{\mathrm{e}}+2 \Omega \int_{V^{\mathrm{e}}} \rho \mathbf{N}^{\mathrm{eT}} \sum_{i=1}^{3} \tilde{u}_{i} \frac{\partial \mathbf{N}^{\mathrm{e}}}{\partial u_{i}} \mathrm{~d} v \dot{\mathbf{q}}^{\mathrm{e}} \\
+\Omega^{2}\left(-\int_{V^{\mathrm{e}}} \rho \mathbf{N}^{\mathrm{eT}} \sum_{i=1}^{3} \mathbf{E} u_{i} \frac{\partial \mathbf{N}^{\mathrm{e}}}{\partial u_{i}} \mathrm{~d} v\right. \\
+\int_{\mathrm{Vol}} \rho \mathbf{N}^{\mathrm{eT}} \sum_{i=1}^{3} \sum_{j=1}^{3} \tilde{u}_{i} \tilde{u}_{j} \frac{\partial^{2} \mathbf{N}^{\mathrm{e}}}{\partial u_{i} \partial u_{j}} \mathrm{~d} v \\
\left.-\int_{\text {Vol }} \rho \mathbf{N}^{\mathrm{eT}} \sum_{i=1}^{3} E u_{i} \mathrm{~d} v\right) \mathbf{q}^{\mathrm{e}}
\end{array}\right) \Phi_{F E}^{\mathrm{e}}
\end{aligned}
$$

Define matrices $\mathbf{M}^{\mathrm{e}}, \mathbf{V}^{\mathrm{e}}, \mathbf{A}^{\mathrm{e}}, \mathbf{C}^{\mathrm{e}}, \mathbf{L}^{\mathrm{e}}$ as follows:

$$
\begin{aligned}
& \mathbf{M}^{\mathrm{e}}=\int_{V^{\mathrm{e}}} \rho \mathbf{N}^{\mathrm{eT}} \mathbf{N}^{\mathrm{e}} \mathrm{d} v ; \\
& \mathbf{V}^{\mathrm{e}}=\int_{V^{\mathrm{e}}} \rho \mathbf{N}^{\mathrm{eT}} \sum_{i=1}^{3} \tilde{u}_{i} \frac{\partial \mathbf{N}^{\mathrm{e}}}{\partial u_{i}} \mathrm{~d} v \\
& \mathbf{C}^{\mathrm{e}}=\int_{V^{\mathrm{e}}} \rho \mathbf{N}^{\mathrm{eT}} \sum_{i=1}^{3} \mathbf{E} u_{i} \frac{\partial \mathbf{N}^{\mathrm{e}}}{\partial u_{i}} \mathrm{~d} v ; \\
& \mathbf{A}^{\mathrm{e}}=\int_{V^{\mathrm{e}}} \rho \mathbf{N}^{\mathrm{eT}} \sum_{i=1}^{3} \sum_{j=1}^{3} \tilde{u}_{i} \tilde{u}_{j} \frac{\partial^{2} \mathbf{N}^{\mathrm{e}}}{\partial u_{i} \partial u_{j}} \mathrm{~d} v ; \\
& \mathbf{L}^{\mathrm{e}}=\int_{V^{\mathrm{e}}} \rho \mathbf{N}^{\mathrm{eT}} \sum_{i=1}^{3} \mathbf{E} u_{i} \mathrm{~d} v ;
\end{aligned}
$$

where matrices $\mathbf{V}^{\mathrm{e}}, \mathbf{A}^{\mathrm{e}}, \mathbf{C}^{\mathrm{e}}, \mathbf{L}^{\mathrm{e}}$ are related to the gyroscopic and inertial effects associated with wheelset rotation.

With 3D solid element, the polynomial shape functions are $\mathrm{C}^{0}$ continuous. Thus, in Eq. (11), the convergence of the integral of matrix $\mathbf{A}^{\mathrm{e}}$ cannot be guaranteed due to the second derivative of the shape function. This problem is solved by integrating $\mathbf{A}^{\mathrm{e}}$ by parts. The integration is taken over all of the three directions in space, which leads to:

$$
\begin{aligned}
\mathbf{A}^{\mathrm{e}}= & \int_{V \mathrm{e}} \rho \mathbf{N}^{\mathrm{eT}} \sum_{i=1}^{3} \sum_{j=1}^{3} \tilde{u}_{i} \tilde{u}_{j} \frac{\partial^{2} \mathbf{N}^{\mathrm{e}}}{\partial u_{i} \partial u_{j}} \mathrm{~d} v \\
= & \int_{S^{\mathrm{e}}} \rho \mathbf{N}^{\mathrm{eT}} \sum_{i=1}^{3} \tilde{u}_{i} \tilde{u}_{1} \frac{\partial \mathbf{N}^{\mathrm{e}}}{\partial u_{i}} \mathrm{~d} u_{2} \mathrm{~d} u_{3}+\int_{S^{\mathrm{e}}} \rho \mathbf{N}^{\mathrm{eT}} \sum_{i=1}^{3} \tilde{u}_{i} \tilde{u}_{2} \frac{\partial \mathbf{N}^{\mathrm{e}}}{\partial u_{i}} \mathrm{~d} u_{3} \mathrm{~d} u_{1} \\
& +\int_{S^{\mathrm{e}}} \rho \mathbf{N}^{\mathrm{eT}} \sum_{i=1}^{3} \tilde{u}_{i} \tilde{u}_{3} \frac{\partial \mathbf{N}^{\mathrm{e}}}{\partial u_{i}} \mathrm{~d} u_{1} \mathrm{~d} u_{2} \\
& -\int_{V^{\mathrm{e}}} \rho\left(\sum_{i=1}^{3} \sum_{j=1}^{3} \tilde{u}_{i} \tilde{u}_{j} \frac{\partial \mathbf{N}^{\mathrm{eT}}}{\partial u_{i}} \frac{\partial \mathbf{N}^{\mathrm{e}}}{\partial u_{j}}-\mathbf{N}^{\mathrm{eT}} \sum_{i=1,3} u_{i} \frac{\partial \mathbf{N}^{\mathrm{e}}}{\partial u_{i}}\right) \mathrm{d} v
\end{aligned}
$$

With Eqs. (8), (10), (11) and (12), the matrices of each element can be assembled into global matrices using a standard finite element method. Consequently, the final motion of equation of the wheelset is:

$$
\begin{aligned}
& \ddot{\mathbf{q}}+2 \Omega \Phi_{F E}^{\mathrm{T}} \mathbf{V} \Phi_{F E} \dot{\mathbf{q}}+\left(\mathbf{K}+\Omega^{2} \Phi_{F E}^{\mathrm{T}}(\mathbf{A}-\mathbf{C}) \Phi_{F E}\right) q \\
& \quad=\Omega^{2} \Phi_{F E}^{\mathrm{T}} \mathbf{L}+\mathbf{Q}
\end{aligned}
$$

where $\mathbf{Q}$ is the generalized forces of the wheelset and $\mathbf{K}$ is the modal stiffness matrix of the finite element wheelset model.

In present work, an ETR500 wheelset profile is modelled (Fig. 1). 3D solid element with 20 nodes is used in the mesh, which can describe the bending motion better than linear solid element. Fifty modes of the wheelset are adopted in the simulation, covering a frequency range up to $1500 \mathrm{~Hz}$ approximately. Some of the mode shapes of the flexible wheelset and the corresponding natural frequencies are reported in Fig. 2 and Table 1. The modal frequencies are validated by experimental data (from [17]) with an acceptable error, see Table 1.

In Fig. 2a, $d$ are mode shapes with multiplicity 1: the first torsional mode and the first umbrella mode. These

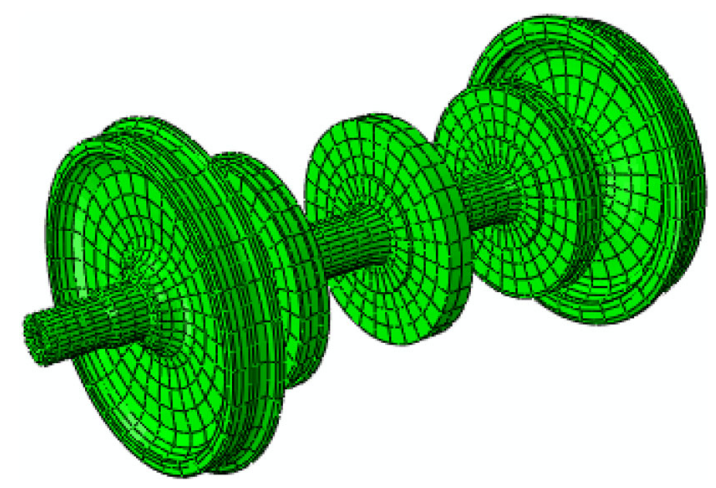

Fig. 1 Finite element model of the wheelset 
Fig. 2 Mode shapes of the flexible wheelset: a first torsional mode; $\mathbf{b}, \mathbf{c}$ first bending mode; $\mathbf{d}$ first umbrella mode; $\mathbf{e}-\mathbf{h}$ first wheelset bending mode

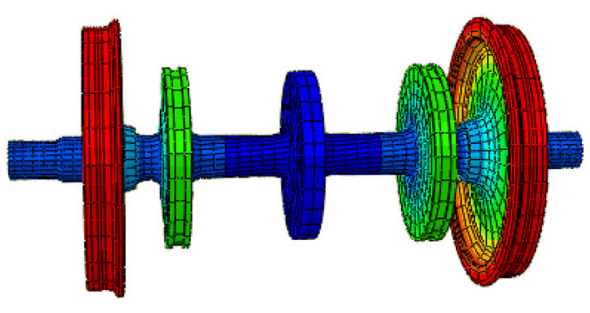

(a)

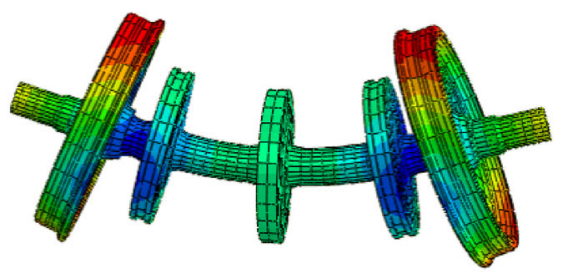

(c)

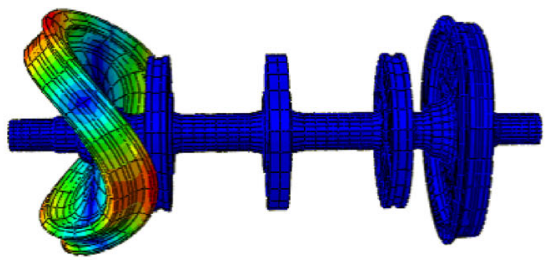

(e)

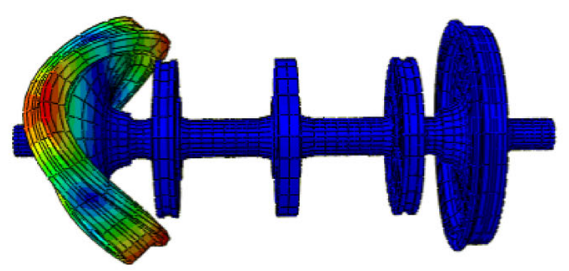

(g)

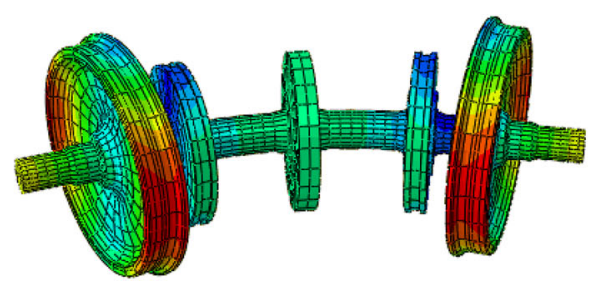

(b)

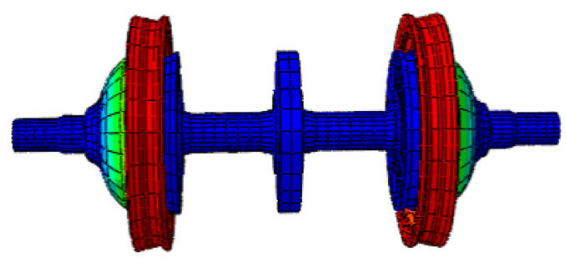

(d)

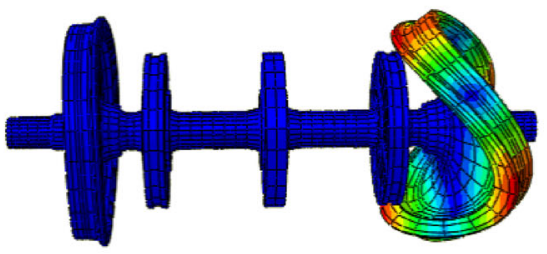

(f)

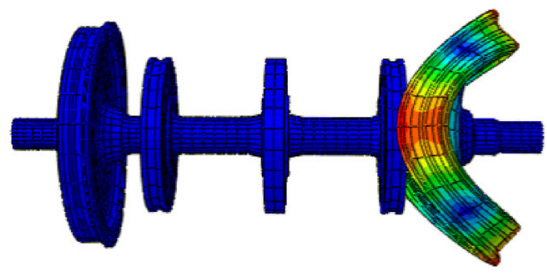

(h) modes are characterized by their deformed shapes having axial symmetry. Modes shown in Fig. 2b, c are examples of modes of multiplicity 2 . They are bending modes of the wheelset axis. For each bending mode, there are two modes with the same natural frequency but their deformed shapes are rotated by a certain angle with respect to each other around the wheelset axis. In Fig. 2e-h the wheel mode shapes with two nodal diameters and no nodal circle are shown. Although the natural frequency corresponding to these mode shapes is the same $(341.3 \mathrm{~Hz})$, they must be regarded as two different modes with multiplicity 2 .

The receptance expression is obtained from Eq. (13):
$H(w)=\boldsymbol{\Phi}_{F E}^{\mathrm{T}}\left[-\omega^{2} \mathbf{I}+2 i \omega \boldsymbol{\Omega} \mathbf{V}+\mathbf{K}+\Omega^{2}((\mathbf{A}-\mathbf{C}))\right] \boldsymbol{\Phi}_{F E}$

Figure 3 shows the vertical receptance of the contact point of the wheelset. For the non-rotating wheelset, the receptance coincides with the natural frequencies of the free-boundary wheelset. If the angular velocity of the wheelset is nonzero, the peaks of the FRF associated with modes with multiplicity 2 (bending modes) produce two peaks that can be interpreted as the forward and backward modes. For instance, when the vehicle velocity is $100 \mathrm{~km} /$ $\mathrm{h}$, the backward mode and the forward mode of the first 
Table 1 Vibration modes of the wheelset

\begin{tabular}{lll}
\hline Vibration mode & $\begin{array}{l}\text { Frequency by } \\
\text { MATLAB (Hz) }\end{array}$ & $\begin{array}{l}\text { Experimental frequency } \\
\text { (Hz) (from [18]) }\end{array}$ \\
\hline First torsional mode & 71.9 & - \\
First vertical and horizontal bending modes (2) & 75.0 & 77 \\
Second vertical and horizontal bending modes (2) & 121.9 & 122 \\
Third vertical and horizontal bending modes (2) & 176.4 & 184 \\
First umbrella mode & 203.3 & 203 \\
Second umbrella mode & 247.3 & 254 \\
Fourth vertical and horizontal bending modes (2) & 317.9 & 315 \\
First bending modes of wheels $(2+2)$ & 341.3 & 335 \\
Fifth vertical and horizontal bending modes (2) & 570.7 & 548 \\
Second bending modes of the wheels $(2+2)$ & 936.8 & 922 \\
\hline
\end{tabular}

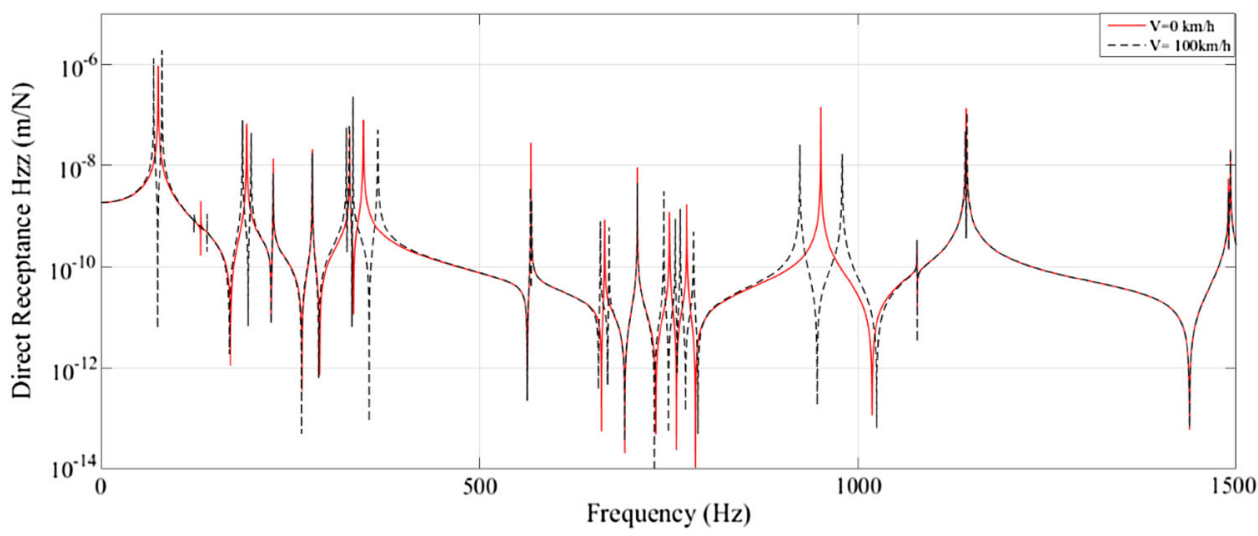

Fig. 3 Direct wheelset receptance at the contact point $\mathrm{Hz}$ for different vehicle velocities bending mode are 65 and $85 \mathrm{~Hz}$, respectively. The peaks of the receptance associated with modes with multiplicity 1 (torsional modes and umbrella modes) remain the same.

A Campbell diagram for the wheelset shows the frequency intersection points of the model. Several intersection points of 'forward' and 'backward' modes of wheelset modes with multiplicity 2 are shown in Fig. 4, which indicates wheelset resonances are affected by the rotation of the wheelset.

\subsection{Model of the Track}

The rail is modelled using Timoshenko beam elements on discrete supports with four elements per sleeper bay. The deformation of the cross section of the rail is neglected in current study since the frequency range interested in the current work is under $1500 \mathrm{~Hz}$ [1]. Half the track only is considered due to the symmetric structure. The half sleepers are modelled as equally spaced masses, while railpads and ballast are modelled as two linear viscoelastic layers with a simple spring-damper model (Fig. 5). A rail with UIC60 section is considered in the track model. The railpad and track bed data come from the EUROBALT project. Parameters are listed in Table 2.

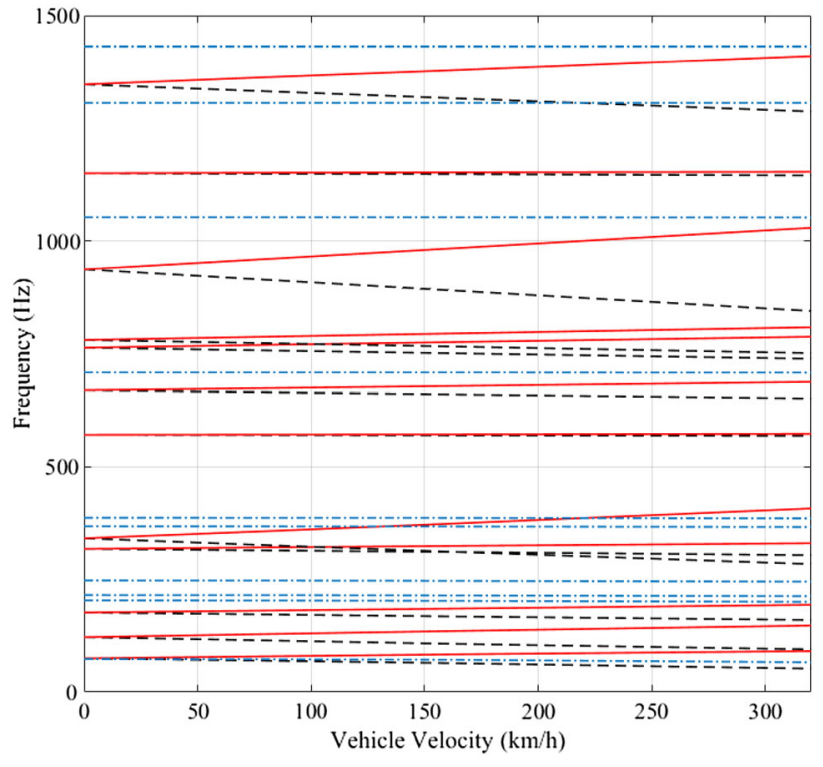

Fig. 4 Campbell diagram for the wheelset

Only horizontal and vertical displacements and angular deflections around horizontal and vertical axes are considered, and motions related to axial and torsional rail 
Fig. 5 Finite element track model

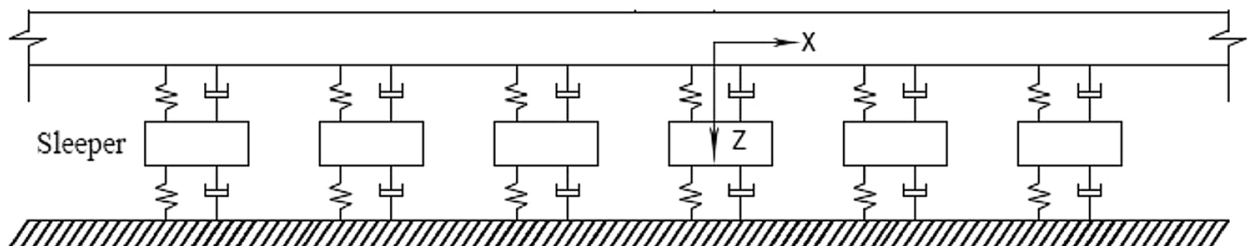

deformation are not included in the analysis. The displacement vector $\left\{\mathbf{u}_{i j}\right\}$ for a general element of the model linking nodes $i$ and $j$ is then

$\left\{\mathbf{u}_{i j}\right\}=\left\{\begin{array}{llllllll}v_{i} & \theta_{i} & u_{i} & \varphi_{i} & v_{j} & \theta_{j} & u_{j} & \varphi_{j}\end{array}\right\}^{\prime}$

The mass and stiffness matrices of the beam elements are determined using Timoshenko beam elements including shear deformation and rotatory inertial effects. The homogeneous Euler-Lagrangian equations for a Timoshenko beam are [18]:

$$
\left\{\begin{array}{l}
k A G\left(v^{\prime \prime}-\theta^{\prime}\right)=0 ; \\
k A G\left(u^{\prime \prime}+\varphi^{\prime}\right)=0 ; \\
k A G \theta+E I_{y} \theta^{\prime \prime}-k A G v^{\prime}=0 ; \\
k A G \varphi+E I_{z} \varphi^{\prime \prime}+k A G u^{\prime}=0 ; \\
k G\left(I_{y}+I_{z}\right) \varphi^{\prime \prime}=0 .
\end{array}\right.
$$

The shape functions of the beam element are formulated by solving the homogeneous Euler-Lagrangian equations with corresponding boundary conditions of the beam element. The stiffness matrix of the rail element is therefore given by:where the coefficients are defined as:
Table 2 Simulation parameters

\begin{tabular}{ll}
\hline Description & Value \\
\hline Wheelset type & ETR500 \\
Wheelset mass & $1375 \mathrm{~kg}$ \\
Axle load & $110 \mathrm{KN}$ \\
Rail type & UIC 60 \\
Sleeper spacing & $0.6 \mathrm{~m}$ \\
Track length & $42 \mathrm{~m}$ \\
Second moment of area of rail & $30.55 \times 10^{-6} \mathrm{~m}^{4}$ \\
Second moment of area of rail & $51.92 \times 10^{-7} \mathrm{~m}^{4}$ \\
Half mass of sleeper & $170 \mathrm{~kg}$ \\
Railpad vertical stiffness & $3 \times 10^{8} \mathrm{~N} / \mathrm{m}$ \\
Railpad vertical damping & $3 \times 10^{4} \mathrm{Ns} / \mathrm{m}$ \\
Railpad lateral stiffness & $2 \times 10^{7} \mathrm{~N} / \mathrm{m}$ \\
Railpad lateral damping & $8 \times 10^{3} \mathrm{Ns} / \mathrm{m}$ \\
Ballast vertical stiffness & $8 \times 10^{7} \mathrm{~N} / \mathrm{m}$ \\
Ballast vertical damping & $8 \times 10^{6} \mathrm{~N} / \mathrm{m}$ \\
Ballast lateral stiffness & $1 \times 10^{5} \mathrm{Ns} / \mathrm{m}$ \\
Ballast lateral damping & $8 \times 10^{3} \mathrm{Ns} / \mathrm{m}$ \\
\hline
\end{tabular}

$$
\mathbf{K}_{r}=\left[\begin{array}{cccccccc}
3 \beta_{z} & 3 a \beta_{z} & 0 & 0 & -3 \beta_{z} & 3 a \beta_{z} & 0 & 0 \\
& a^{2} \beta_{z}\left(4+3 \alpha_{z}\right) & 0 & 0 & -3 a \beta_{z} & a^{2} \beta_{z}\left(2-3 \alpha_{z}\right) & 0 & 0 \\
& & 3 \beta_{y} & -3 a \beta_{y} & 0 & 0 & -3 \beta_{y} & -3 a \beta_{y} \\
& & & a^{2} \beta_{y}\left(4+3 \alpha_{y}\right) & 0 & 0 & 3 a \beta_{y} & a^{2} \beta_{y}\left(2-3 \alpha_{y}\right) \\
& & & & 3 \beta_{z} & -3 a \beta_{z} & 0 & 0 \\
& & & & & a^{2} \beta_{z}\left(4+3 \alpha_{z}\right) & 0 & 0 \\
& & & & & & 3 \beta_{y} & 3 a \beta_{y} \\
& & & & & & a^{2} \beta_{y}\left(4+3 \alpha_{y}\right)
\end{array}\right]
$$

$$
\begin{gathered}
\alpha_{z}=\frac{E I_{z}}{k A G a^{2}}, \quad \beta_{z}=\frac{E I_{z}}{2 a^{3}\left(1+3 \alpha_{z}\right)}, \quad \alpha_{y}=\frac{E I_{y}}{k A G a^{2}}, \\
\beta_{y}=\frac{E I_{y}}{2 a^{3}\left(1+3 \alpha_{y}\right)}
\end{gathered}
$$

being $E$ the Young's modulus, $I$ the second moment of area, $A$ the cross-sectional area, $k$ the Timoshenko shear coefficient, $G$ the shear modulus, and $a=0.075 \mathrm{~m}$ is half the element length.

A Rayleigh damping is used for the rail, with a corresponding damping ratio $0.6 \%$. Mass, stiffness and damping matrix for the railpad element (including the mass of the sleeper) and ballast element are set as two-layer parallel 


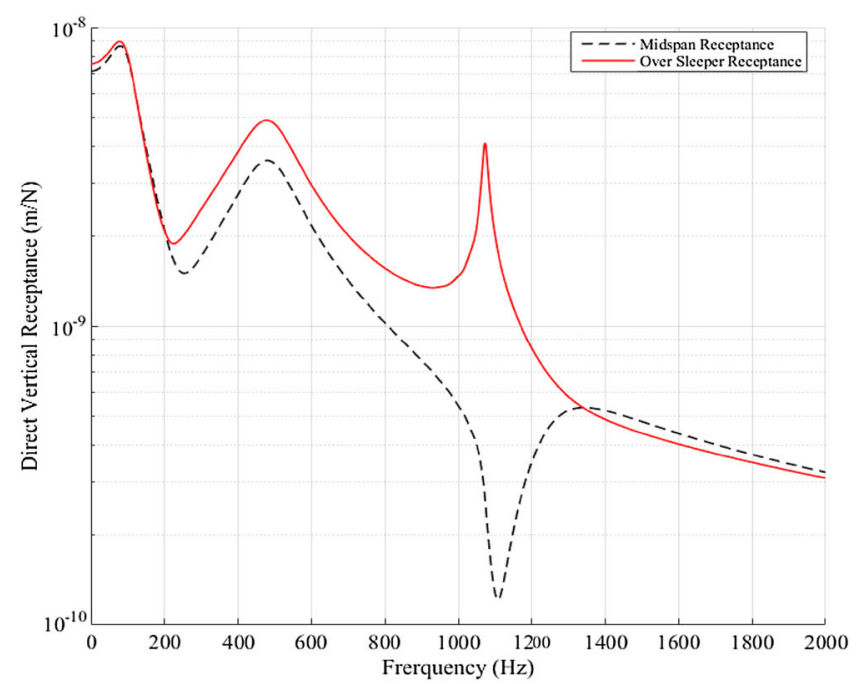

(a)

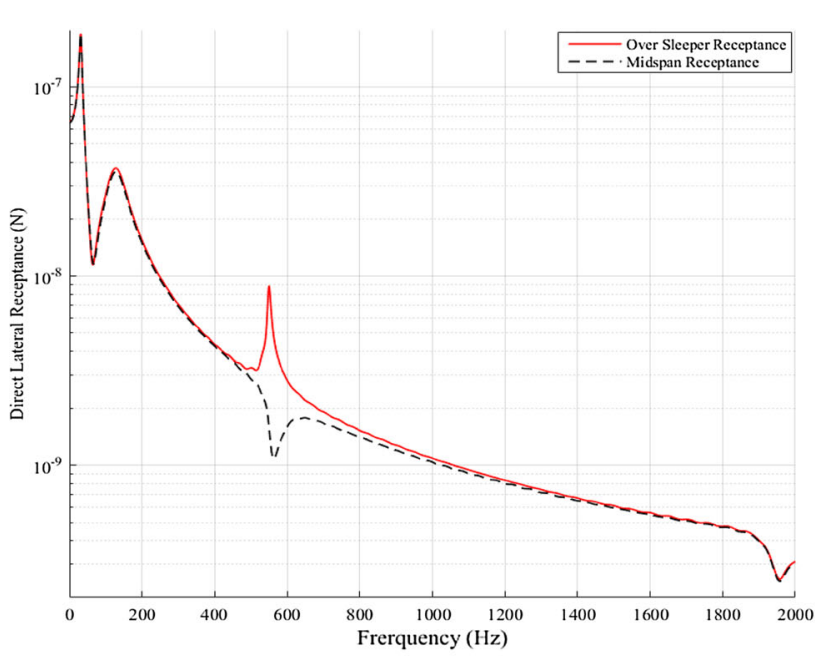

(b)

Fig. 6 Track receptance: a direct receptance of the vertical direction; $\mathbf{b}$ direct receptance of the lateral direction

spring-dashpot elements with the ground nodes being clamped constrained. A set of equations of motion for the track is therefore written in a matrix form:

$\mathbf{M}_{t} \ddot{\mathbf{u}}_{t}+\mathbf{C}_{t} \dot{\mathbf{u}}_{t}+\mathbf{K}_{t} \mathbf{u}_{t}=\mathbf{F}_{c t}$

where $\mathbf{M}_{t}, \mathbf{C}_{t}$ and $\mathbf{K}_{t}$ are the mass, damping and stiffness matrix of the track model, respectively. $\mathbf{F}_{c t}$ is the generalized force applied on the contact point of the rail surface.

The direct receptance of the track in both vertical and lateral direction is obtained (cf. Fig. 6), and the results match with that obtained in previous study [4], which is proved to be valid for dynamic study of frequency up to $1500 \mathrm{~Hz}$.

\subsection{Model of Wheel-Rail Contact Force}

The nonlinear normal force between wheel and rail surface is solved using Hertz's theory [19]:

$F_{n}=C_{h} \delta^{1.5}$

where the constant $C_{h}$ depends from the contact surfaces bending and from material characteristics and $\delta$ is the value of the elastic penetration between the two bodies.

Tangential and lateral forces have been calculated according to Kalker's linear theory [20]:

$$
\begin{aligned}
& \varepsilon_{\mathrm{t}}=\frac{\left(\dot{x}_{c w}-\dot{x}_{c t}\right)}{V}, \quad \varepsilon_{l}=\frac{\left(\dot{y}_{c w}-\dot{y}_{c t}\right)}{V} ; \\
& F_{t}=f_{11} \cdot \varepsilon_{t}, \quad F_{l}=f_{22} \cdot \varepsilon_{l}
\end{aligned}
$$

where $V$ represents the speed of the relative reference with respect to an absolute observer (i.e. the wheelset speed) and $f_{11}$ and $f_{22}$ are constants dependent on dimensions of the contact area and the elastic material properties. Since a motion of the wheelset on a straight path at consistent speed has been taken into account in the present model, the creepages are very little, which meets the hypothesis of Kalker's linear theory. The ODE45 routine based on an explicit Runge-Kutta formula in MATLAB is used to solve simultaneously the rail and the wheel equations.

\section{Results and Discussion}

This section presents the results of simulations under different rail irregularities: a random rail corrugation and several single-harmonic corrugations. The simulation is to investigate the influence of different track and wheelset modelling options on wheel-rail contact forces, which are important to wear and damage phenomena in the rolling surfaces.

\subsection{Response to Random Rail Corrugation}

In order to investigate how the corrugation wavelength may influence the results from the track model and the wheelset models, simulations are carried out when the rail roughness is described by a white-noise band function. Roughness is defined as an additional component of the relative displacement between the wheel and the rail. The amplitude of the random corrugation is $0.1 \mu \mathrm{m}$, and the wavelengths were chosen between 2.7 and $67.7 \mathrm{~cm}$, covering the frequency range from 60 to $1500 \mathrm{~Hz}$ if the vehicle velocity is $40 \mathrm{~m} / \mathrm{s}$.

Figure 7 shows the contact force of three directions for a flexible track and an infinitely rigid track, respectively. A rotating flexible wheelset model was used for both 

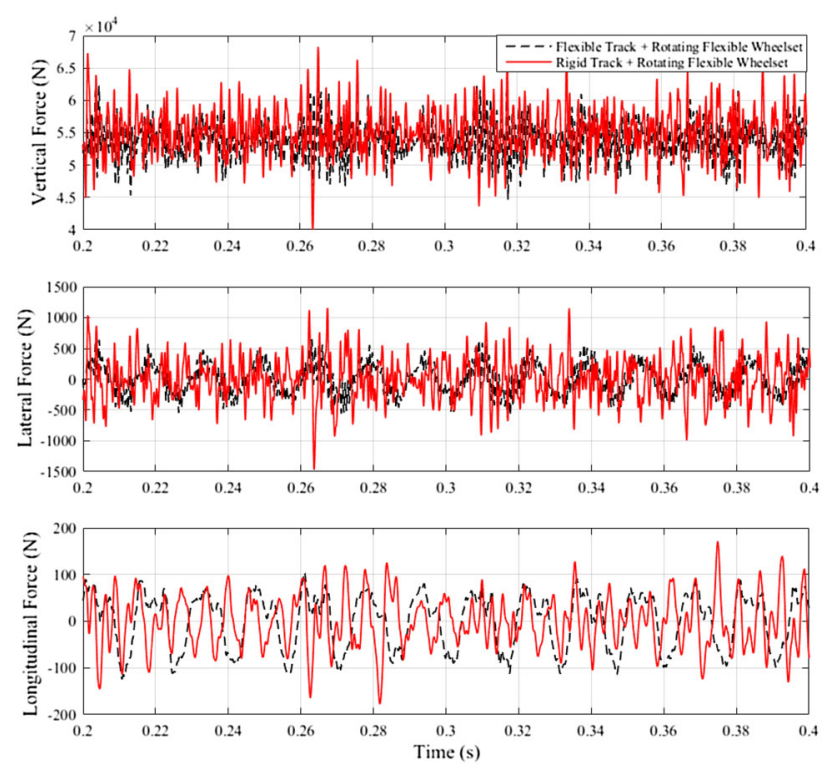

Fig. 7 Contact forces for different track options

interaction models. With a flexible track, a periodic vibration component corresponding to the sleeper bay could be seen from the three directions. To investigate the difference caused by track flexibility, a discrete Fourier transform modulus of the contact forces was taken (Fig. 8). Four peaks can be observed at frequency 67, 134, 201 and $268 \mathrm{~Hz}$ for the flexible track model, which is due to the sleeper bay and the length of each beam element of the track. In the frequency range below $800 \mathrm{~Hz}$, the spectra of the contact forces are on average higher for the model considering a rigid track, compared to the model considering a flexible track. This is due to the reduction in impedance produced in this frequency range by track
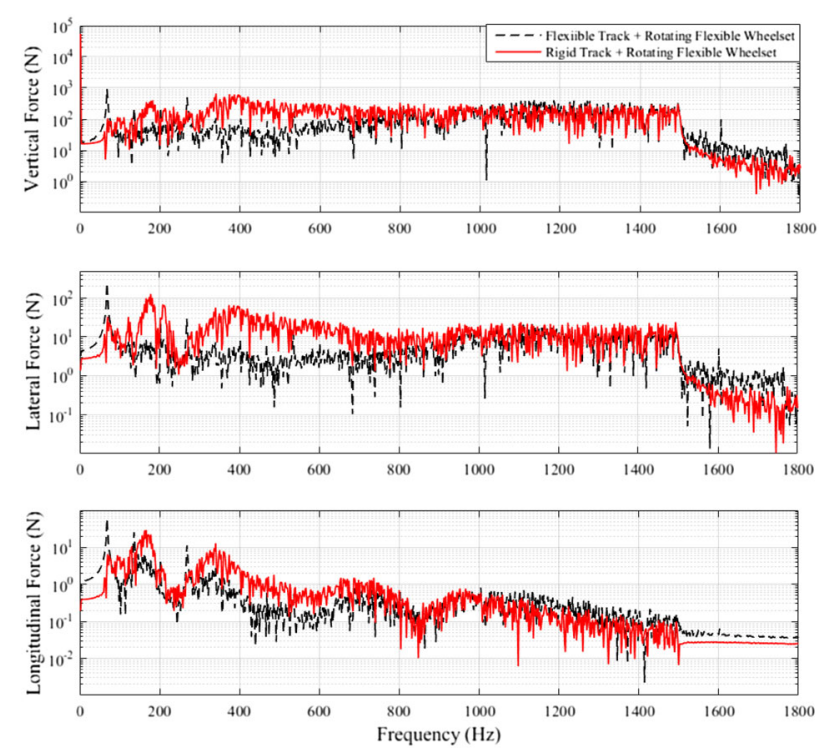

Fig. 8 Spectra of the contact forces for different track options flexibility. For frequency higher than $1000 \mathrm{~Hz}$, the flexibility of the track leads to slightly higher amplitude of both vertical and longitudinal forces.

Figure 9 shows the contact force of three directions with a non-rotating flexible wheelset and a rotating flexible wheelset. A flexible track model was used for both interaction models. The time histories of the vertical and lateral contact forces of the two different wheelset models are almost the same, while the longitudinal force of the rotating flexible wheelset shows a higher amplitude with the non-rotating one. Actually, the longitudinal component of the non-rotating flexible wheelset is very small, with a max value around $25 \mathrm{~N}$, i.e. 200 times smaller than the amplitude of the dynamic fluctuation of the vertical force. Considering wheelset rotation instead, a small longitudinal force is generated, due to the effect of gyroscopic forces on the modes of the rotating wheelset involving axle bending in the horizontal plane. Despite the fact the force amplitude is still small compared to the vertical and lateral force components, it shows a big difference from the case of the non-rotating wheelset.

The Fourier transform modulus of the vertical and lateral forces (Fig. 10) shows slight difference with non-rotating and rotating flexible wheelset. For longitudinal force, the rotating flexible wheelset shows a higher component than the non-rotating flexible wheelset under $800 \mathrm{~Hz}$. The peak frequencies of rotating flexible wheelset can be found to correspond with the forward and backward mode frequencies at $40 \mathrm{~m} / \mathrm{s}$. For instance, the first two peak frequencies of rotating flexible wheelset appear at 67 and $135 \mathrm{~Hz}$. At $67 \mathrm{~Hz}$, the peak meets the backward first bending mode and the frequency due to the sleeper bay. At
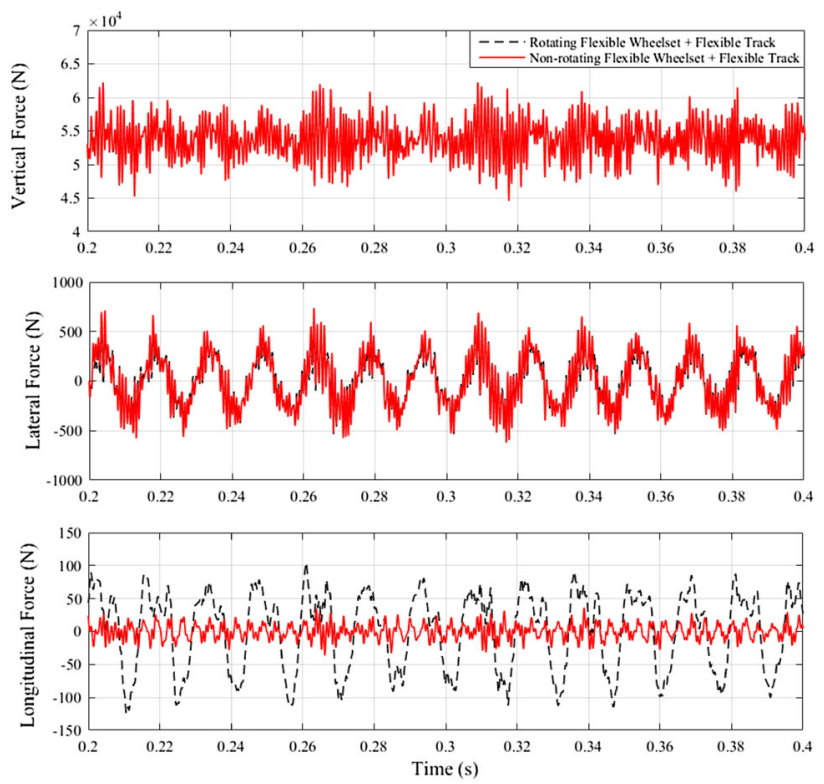

Fig. 9 Contact forces of the wheelset running on randomly worn rails 

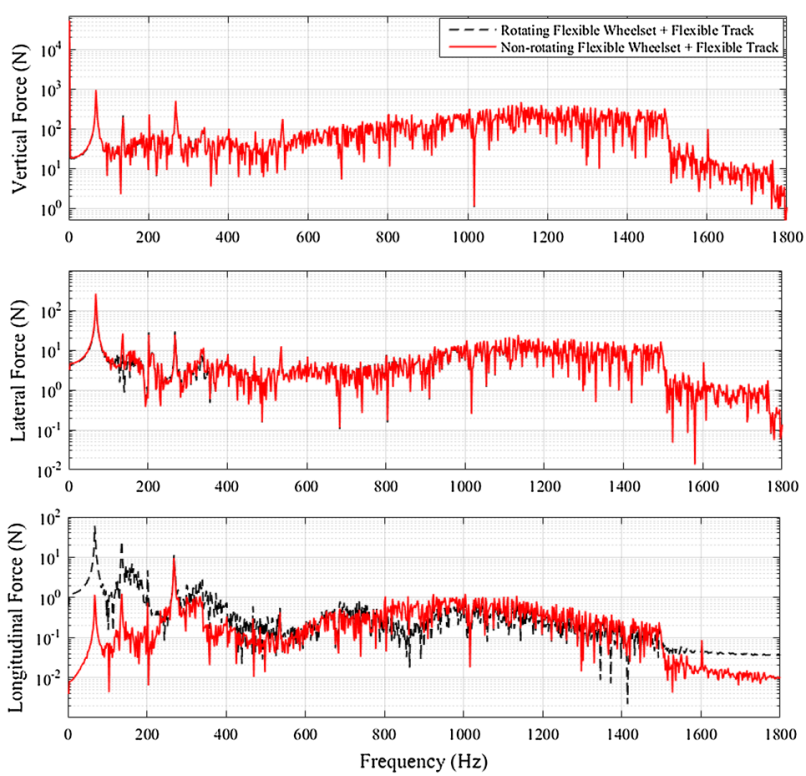

Fig. 10 Spectra of the contact forces for different wheelset options

about $135 \mathrm{~Hz}$, the peak appears due to the forward second bending mode of the wheelset and the frequency due to the mid-sleeper bay.

\subsection{Response to Single-Harmonic Rail Corrugation}

In order to quantify the relevance of dynamic effects affecting the wheel-rail contact forces, the peak values for the vertical component of the contact forces normalized with respect to the static wheel load are reported with increasing vehicle speed under several single-harmonic rail corrugations which may excite the resonances of the wheelset and the track. Two kinds of harmonic excitation are chosen: the first one has an excitation frequency equal to the pinned-pinned frequency of the finite element track, and the second one coincides with the fifth backward mode of the rotating wheelset.

Figure 11 shows the dynamic ratio of the vertical contact force peak value for an excitation at pinned-pinned frequency with different track models. For both models, the dynamic ratio increases with increasing velocity. For the flexible track model, the ratio is $10-15 \%$ higher than the rigid track and shows a greater increasing trend compared to the rigid one, confirming the influence of the flexible track model.

Figure 12 shows the ratio of peak value of the vertical contact force to the static wheel load with different wheelset models. A rigid wheelset is added to this analysis. The fifth backward mode of the rotating wheelset is chosen as the excitation frequency to investigate influence of the resonance frequencies of the rotating wheelset, which is shown as intersection points at $322 \mathrm{~Hz}$ with a velocity of

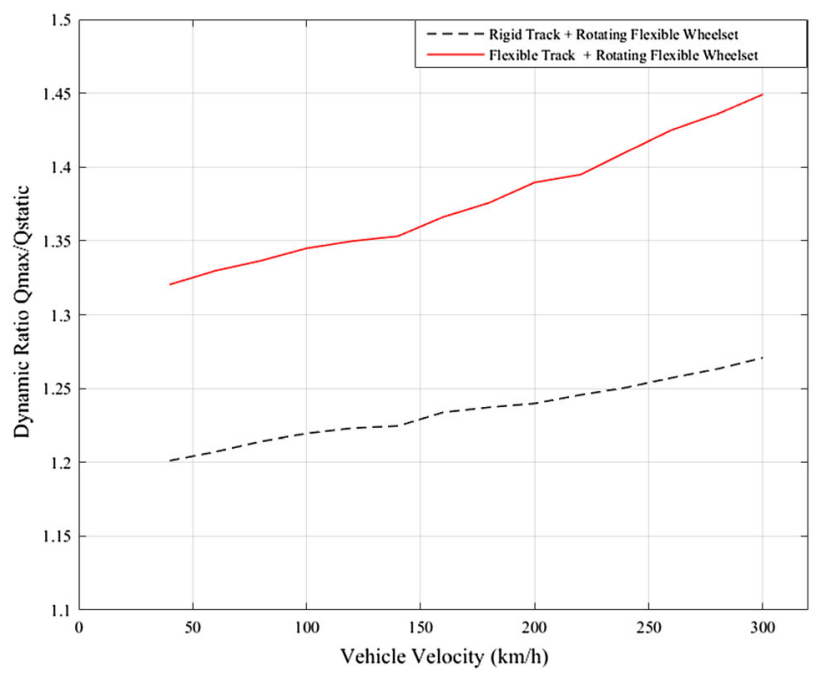

Fig. 11 Dynamic ratio of different track models for an excitation at pinned-pinned frequency

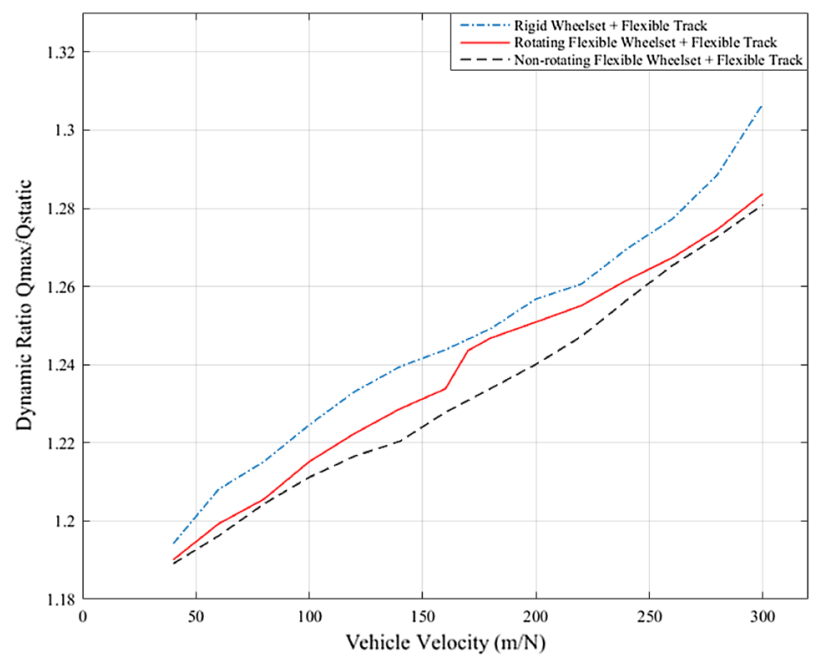

Fig. 12 Dynamic ratio of different track models for an excitation at the fifth backward frequency of the rotating wheelset

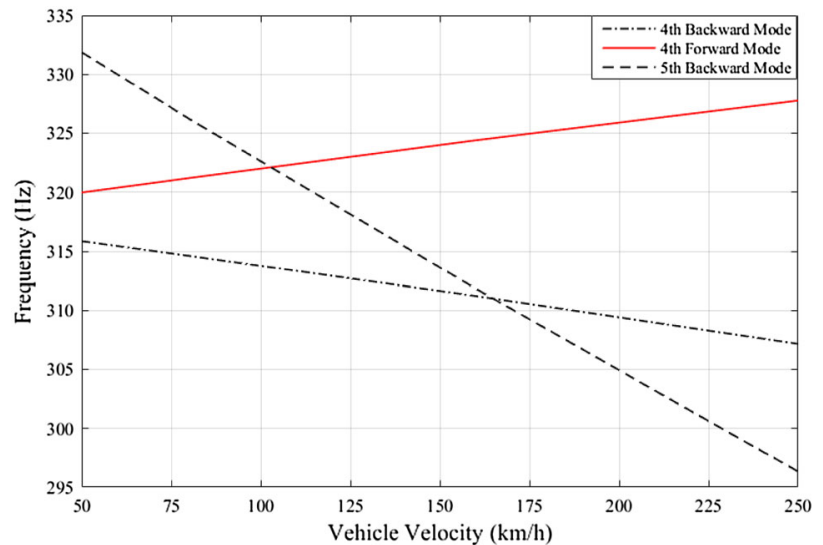

Fig. 13 Intersection points for the fifth bending backward mode of the rotating wheelset 
$103 \mathrm{~km} / \mathrm{h}$ and at $311 \mathrm{~Hz}$ with a velocity of $165 \mathrm{~km} / \mathrm{h}$ (Fig. 13). It is observed that the average dynamic ratio is higher for the model considering a rigid wheelset than for the model accounting for wheelset flexibility. This is due to a compensation of the wheelset flexibility to the contact forces. For the flexible wheelset model, the dynamic ratio of the rotating flexible wheelset shows a slightly higher amplitude with the non-rotating flexible wheelset. A peak ratio at around $165 \mathrm{~km} / \mathrm{h}$ for the rotating wheelset can be found, which is the resonance frequency related to the intersection point of the fifth backward and the fourth backward bending modes, while the intersection point at $103 \mathrm{~km} / \mathrm{h}$ does not show significant amplification of the dynamic contact force.

\section{Conclusion}

This paper proposed a vehicle-track interaction model in time domain, which is valid for dynamic simulation up to $1500 \mathrm{~Hz}$. The effect of wheelset and track flexibility on dynamics of wheelset-track interaction was investigated.

Results of the contact forces under different structure flexibilities are presented for two kinds of rail corrugation: a randomly corrugated track and several single-harmonic corrugated tracks. For the random corrugation, the Timoshenko beam track model shows obvious difference compared with a rigid track model. Force peaks on the three directions could be found in the discrete support flexible track at each sleeper bay position. In the frequency range below $800 \mathrm{~Hz}$, the flexible track model shows lower contact forces than the rigid model, while for frequency higher than $1000 \mathrm{~Hz}$, the contact forces of the flexible track model are slightly higher than for the rigid track model. The rotating flexibility of the wheelset produces a higher longitudinal force when compared with a non-rotating flexible wheelset, while the vertical and lateral forces show little difference between the two wheelset models. For the single-harmonic corrugation case, the numerical results show that the wheel-rail contact force amplitude is generally increasing with growing velocities and shows a peak when the frequency of excitation meets the resonance frequencies of the flexible structure. However, it must be highlighted that the effect of velocity is more remarkable.

Overall, the results presented above show that simulation results are highly sensitive to the track model and wheelset model adopted. Neglecting wheelset rotating flexibility and track flexibility may lead to an overestimation or underestimation of the dynamic contact forces generated by the vehicle in response to track imperfections, depending on the type of excitation and on the frequency range considered.
The model in this paper is valid up to $1500 \mathrm{~Hz}$ in wheel-rail interaction analysis, which can be used for investigation of specific interaction problems, e.g. rail short-pitch corrugation. However, for problems related to higher-frequency range, e.g. rolling noise problem, the model of the track still needs to be improved. Future developments of this work will be addressed to further expanding the frequency range of validity of the train-track interaction model, by introducing a more detailed track model based on solid FEM in a moving reference.

Acknowledgements This work has been financially supported by the China Scholarship Council.

Open Access This article is distributed under the terms of the Creative Commons Attribution 4.0 International License (http://crea tivecommons.org/licenses/by/4.0/), which permits unrestricted use, distribution, and reproduction in any medium, provided you give appropriate credit to the original author(s) and the source, provide a link to the Creative Commons license, and indicate if changes were made.

\section{References}

1. Thompson D (2008) Railway noise and vibration: mechanisms, modelling and means of control. Elsevier, London

2. Sun W, Zhou J, Thompson D, Gong D (2014) Vertical random vibration analysis of vehicle-track coupled system using Green's function method. Veh Syst Dyn 52(3):362-389

3. Thompson D, Jones C (2000) A review of the modelling of wheel/rail noise generation. J Sound Vib 231(3):519-536

4. Knothe K, Grassie S (1993) Modelling of railway track and vehicle/track interaction at high frequencies. Veh Syst Dyn 22(3-4):209-262

5. Arnold J, Kaiser I, Schupp G (2005) Simulation of a railway vehicle's running behaviour: how elastic wheelsets influence the simulation results. Veh Syst Dyn 41:242-251

6. Gómez J, Vadillo E, Santamaría J (2006) A comprehensive track model for the improvement of corrugation models. J Sound Vib 293(3):522-534

7. Kaiser I, Popp K (2006) Interaction of elastic wheelsets and elastic rails: modelling and simulation. Veh Syst Dyn 44(sup1):932-939

8. Baeza L, Fayos J, Roda A, Insa R (2008) High frequency railway vehicle-track dynamics through flexible rotating wheelsets. Veh Syst Dyn 46(7):647-659

9. Zhai W, Wang K (2010) Lateral hunting stability of railway vehicles running on elastic track structures. J Comput Nonlinear Dyn 5(4):1-9

10. Baeza L, Ouyang H (2011) A railway track dynamics model based on modal substructuring and a cyclic boundary condition. J Sound Vib 330(1):75-86

11. Baeza L, Vila P, Xie G, Iwnicki SD (2011) Prediction of rail corrugation using a rotating flexible wheelset coupled with a flexible track model and a non-Hertzian/non-steady contact model. J Sound Vib 330(18):4493-4507

12. Di Gialleonardo E, Braghin F, Bruni S (2012) The influence of track modelling options on the simulation of rail vehicle dynamics. J Sound Vib 331(19):4246-4258

13. Kaiser I (2012) Refining the modelling of vehicle-track interaction. Veh Syst Dyn 50(sup1):229-243 
14. Martínez-Casas J, Mazzola L, Baeza L, Bruni S (2013) Numerical estimation of stresses in railway axles using a train-track interaction model. Int J Fatigue 47:18-30

15. Shabana AA (2013) Dynamics of multibody systems. Cambridge University Press, Cambridge

16. Brown M, Shabana A (1997) Application of multibody methodology to rotating shaft problems. J Sound Vib 204(3):439-458

17. Francesco R, Roberto A, Bruni S (2007) A time domain model for the study of high frequency train-track interaction. Paper presented at the 7th international conference on railway bogies and running gears, Budapest

18. Petyt M (2010) Introduction to finite element vibration analysis. Cambridge University Press, Cambridge

19. Johnson KL, Johnson KL (1987) Contact mechanics. Cambridge University Press, Cambridge

20. Kalker JJ (1990) Three-dimensional elastic bodies in rolling contact, vol 2. Kluwer, Amsterdam 